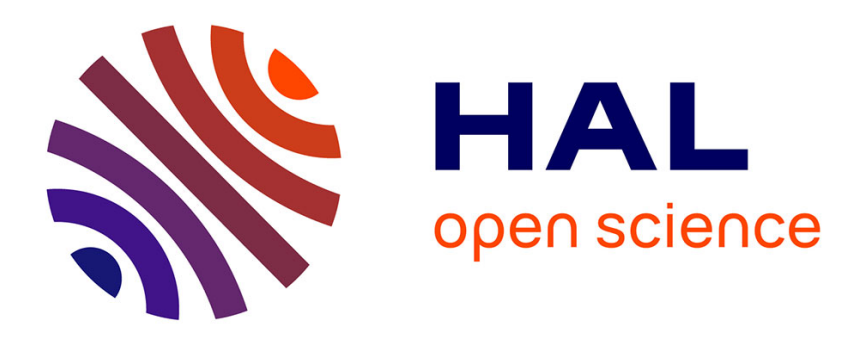

\title{
Sticking collision between a sphere and a textured wall in a viscous fluid
}

Thibault Chastel, Anne Mongruel

\section{To cite this version:}

Thibault Chastel, Anne Mongruel. Sticking collision between a sphere and a textured wall in a viscous fluid. Physical Review Fluids, 2019, 4 (1), pp.014301. 10.1103/PhysRevFluids.4.014301. hal-02089710

\section{HAL Id: hal-02089710 https://hal.sorbonne-universite.fr/hal-02089710}

Submitted on 5 Apr 2019

HAL is a multi-disciplinary open access archive for the deposit and dissemination of scientific research documents, whether they are published or not. The documents may come from teaching and research institutions in France or abroad, or from public or private research centers.
L'archive ouverte pluridisciplinaire HAL, est destinée au dépôt et à la diffusion de documents scientifiques de niveau recherche, publiés ou non, émanant des établissements d'enseignement et de recherche français ou étrangers, des laboratoires publics ou privés. 


\title{
Sticking collision between a sphere and a textured wall in a viscous fluid
}

\author{
Thibault Chastel and Anne Mongruel* \\ Physique et Mécanique des Milieux Hétérogènes, \\ ESPCI Paris, PSL University, CNRS, Sorbonne Université, \\ Univ. Paris Diderot, Sorbonne Paris Cité, 75005 Paris France.
}

The collision of a sphere with a wettable micro-textured wall in a viscous fluid is investigated experimentally, focusing on the region close to the contact with the wall. High frequency laser interferometry is used for measuring small displacements of the sphere in that region. The wall texture consists of an a array of square micro-pillars, whose geometrical parameters (height, width and spacing of the pillars) are varied. The wall texture decreases the hydrodynamic resistance, and hence the drag on the sphere, compared to the case of a smooth wall. At small Reynolds and Stokes numbers, this drag reduction is quantified in terms of an equivalent plane boundary, shifted down from the top of the pillars. The shift length depends on the geometrical parameters of the pillars array, and is compared to available predictions of effective slip length for a flow over arrays of micro-pillars in the Wenzel state. At finite Stokes number, below the bouncing transition, the wall texture influences the relative importance of sphere inertia and drag force in the near wall region. As a result, a great diversity of sphere dynamics are obtained by varying the texture geometrical parameters. These dynamics can be captured considering a shift-length-modified drag force in the equation of motion of the sphere.

\footnotetext{
* anne.mongruel@sorbonne-universite.fr
} 


\section{INTRODUCTION}

Particle collisions with walls or with other particles in a fluid play an important role in many natural or industrial processes involving particle-laden flows, such as erosion of particle beds, filtration, or sedimentation. From a fundamental point of view, particle-particle or particle-wall hydrodynamic interactions are crucial to the understanding of the dynamics of concentrated suspensions, or wet granular media. The near-contact motion of two approaching particles is opposed by the drainage of the fluid film between them, which slows down their approach. The lubrication force on the particles due to the large pressures developed in the fluid being squeezed out diverges as the gap between the particles reduces. If the particle inertia is not sufficient, the relative velocity eventually vanishes, and physical contact occurs when the distance between the surface decreases to the height of the roughness of the real surfaces $([1],[2])$. In the opposite case, the viscous force resisting the approach is not sufficient to slow their approach, and the particles collide with a nonzero velocity. Moreover, if the materials are elastic, a portion of the kinetic energy of the particles is stored as elastic deformation energy, and it may happen that following contact, bouncing then occurs.

Resolving the question of an immersed collision with or without bouncing is a longstanding problem in which fluid forces, particle inertia, and elastic deformation of the particles participate. The Stokes number, defined as the ratio of particle inertia to viscous forces, was predicted to be the parameter controlling the transition from sticking to bouncing collision ([3]). This was verified experimentally, in the case of a sphere released in air towards a wall covered with a thin layer of viscous liquid ([4], [5]), or in the case of a sphere fully immersed in a viscous liquid and settling towards a wall $([6],[7],[8])$. In those studies, no bouncing was occuring below a critical value of the Stokes number, $S t_{c} \simeq 10$. Interestingly, experimental works also shown that the presence of roughness on the contacting surfaces had a significant effect on promoting rebound ([4], [7]). Indeed, it appears that the surface roughness is of outmost importance to the whole collision process. As shown in recent experimental ([9]), numerical ([10], [11]) and analytical works ([12]), the surface roughness influences the different steps of the collision, from the dynamics of approach of the sphere close to the wall, to the bouncing dynamics and subsequent height of rebound.

The present work investigates in details how near-wall hydrodynamic interactions between a sphere and a textured wall may slow down the particle to such an extent as to hinder the bouncing. The Stokes numbers considered here is sub-critical, but with values close to the bouncing transition. The sphere is smooth, millimetric, and settles in a viscous fluid towards a textured wall. The texture 
stands for a model roughness : it consists of an array of square micro-pillars, whose geometrical parameters can be easily controlled using standard micro-fabrication techniques. The pillars are completely impregnated by the fluid (Wenzel state). The pillars of width $2 b$ and height $e$ are organized in a periodic square pattern of period $L$. The values of these geometrical parameters are of the order of $10 \mu \mathrm{m}$, and are varied systematically in order to study their influence on the sphere dynamics. The characteristic period of the array, $L$, is small compared to the sphere radius, $a$. The region of interest is the near-wall region, where the gap $h$ between the sphere and the top of the pillars is small compared to the sphere radius, here typically smaller than a millimeter. Only a few experimental data are available in that region ([13]) because of the limited spatial resolution of most high-speed video cameras. To resolve the dynamics of the sphere in time and space, we use a high frequency interferometric device, which already proved useful for studying the dynamics of sphere near a smooth wall at finite Stokes numbers below $S t_{c}([14])$, and near a textured wall at vanishing Stokes number ([15], [16], [17]). Recently, we measured with the same device the contact dynamics and the micro-rebounds of a sphere on a textured wall ([9]). In this latest work, we shown that the bouncing transition was critically influenced by the geometrical parameters of the texture. The present article aims to supplement the picture of the collision process by presenting new experimental results on the dynamics of a sphere approaching a textured wall at finite Stokes numbers below $S t_{c}$. The novelty of the present study is to fill a lack of experimental data in this often neglected regime close to the bouncing transition. Moreover, it reveals the diversity of dynamics that can be obtained in this regime by finely tuning the geometrical parameters of the texture. Finally, it provides a quantitative understanding of the effect of a wall micro-texture on the sticking collision.

In a first step, we characterize the hydrodynamic effect of the wall texture at small Stokes and Reynolds numbers. This effect amounts to a drag reduction on the sphere compared to the case of a smooth wall that would be located at the top of the pillars. We notice that this drag reduction is spatially varying. At distances from the wall large compared to the period of the pillar array $(h \gg L)$, the drag reduction can be quantified using the notion of an equivalent plane boundary, shifted down from the top of the pillars $([15],[18])$. We show that the corresponding "far-field" shift lengths depends on the texture geometrical parameters. This dependency is compared to scaling laws for effective slip length originally introduced in the context of superhydrophobic slippage but also applicable to the case of a liquid-impregnated texture ([19]). To quantify the drag reduction at small distances $(h \ll L)$, we introduce a "near-field" shift length for each texture that will be useful in the second part of the study. 
In a second part, we study how the wall texture influences the dynamics of approach of the sphere at Stokes numbers close to (but below) the bouncing transition, i.e. in the case of a sticking collision. We present the different sphere dynamics obtained by varying the Stokes number and the texture geometrical parameters. To describe these dynamics, a model is developed using a drag force modified by the near-field shift lengths introduced in the first part. Finally, we discuss how these findings may be used to predict the transition from sticking to bouncing collision.

\section{EXPERIMENTAL METHODS}

\section{A. Particles and fluids}

The sphere settles in a fluid contained in a cylindrical container with a $50 \mathrm{~mm}$ diameter and a $40 \mathrm{~mm}$ height. The lateral walls are made with altuglass, and the top and bottom plane walls are made of glass of optical quality. The fluid is a silicon oil (Bluestar) of density $\rho_{f}=978 \mathrm{~kg} / \mathrm{m}^{3}$. For experiments at small and moderate Reynolds number respectively, we used two oil grades $47 \mathrm{~V} 100000$ and 47V1000 whose kinematic viscosities at $25^{\circ} \mathrm{C}$ are $\nu=10^{-1} \mathrm{~m}^{2} / \mathrm{s}$ and $\nu=10^{-3}$ $\mathrm{m}^{2} / \mathrm{s}$ respectively. The oil viscosity and refraction index $\left(n=1.404\right.$ at $\left.25^{\circ} \mathrm{C}\right)$ vary slowly with temperature. The oils have a Newtonian behaviour for shear-rates up to $100 \mathrm{~s}^{-1}$ and $2500 \mathrm{~s}^{-1}$ respectively. We have estimated the magnitude of the shear-rates created in the squeezed fluid before the collision, and found that they stay below the values for the onset of shear-thinning ([9]). The particles are spherical balls with diameters ranging from $7 \mathrm{~mm}$ to $15.9 \mathrm{~mm}$. The sphere material is either steel (density $\rho_{p}=7.8 \times 10^{3} \mathrm{~kg} / \mathrm{m}^{3}$, elastic modulus $E_{p}=240 \mathrm{GPa}$ ) or tungsten carbide (density $\rho_{p}=15.6 \times 10^{3} \mathrm{~kg} / \mathrm{m}^{3}$, elastic modulus $E_{p}=550 \mathrm{GPa}$ ). The roughness $R_{a}$, as provided by the manufacturer, is of $0.1 \mu \mathrm{m}$ for the steel balls, and $0.013 \mu \mathrm{m}$ for the tungsten carbide ball.

The particle Stokes number is defined as $S t=(2 / 9) \rho_{p} a V_{T} / \eta$ where $\eta=\rho_{f} \nu$ is the dynamic viscosity of the oil and $V_{T}$ is a characteristic settling velocity of the sphere. We use for $V_{T}$ the terminal velocity of the sphere in the same unbounded fluid. The corresponding particle Reynolds number is defined as $R e=(2 a) V_{T} / \nu=9\left(\rho_{f} / \rho_{p}\right) S t$. For small values of this Reynolds number, $V_{T}$ coincides with the Stokes velocity $V_{S t}=(2 / 9)\left(\rho_{p}-\rho_{f}\right) a^{2} / g$. For finite Reynolds number $V_{T}$ is calculated using the Oseen correction for small inertia effects on the steady drag([14]). Values of $V_{T}, R e$ and $S t$ are summarized in table II in the case of spheres of different diameters settling in the 47V1000 silicon oil. Note that in our experiments, Re and St are of the same order of magnitude. 

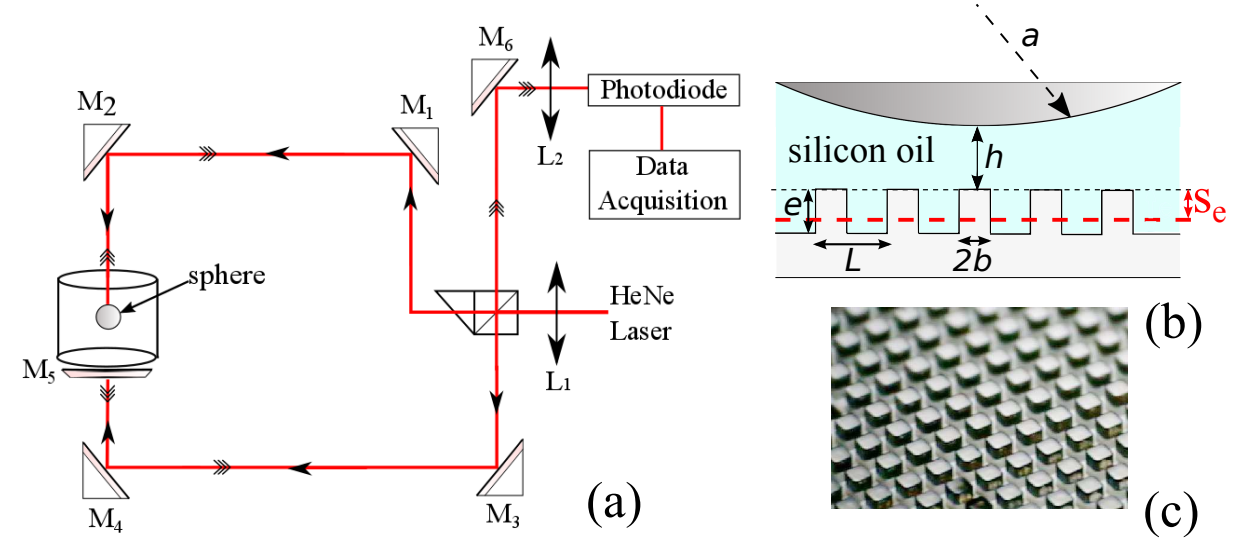

FIG. 1. Schematic of the experimental set-up. (a) settling sphere interferometer (b) side view of a sphere near an array of micro-pillars, and position of the equivalent smooth plane (c) Profilometry of a micro-pillar array with $\Phi=0.3, L=179 \mu \mathrm{m}, 2 b=106 \mu \mathrm{m}$, and $e=85 \mu \mathrm{m}$.

\section{B. Textured surfaces}

Wettable textured surfaces are made in a clean room using standard lithography. Geometric shapes are obtained by soft imprint of a photo-curable polymer, a thiolene based resin (NOA 81, Norland optical adhesives) on glass microscope slides to be fixed at the bottom wall of the container. The resin is oleophilic so that the oil completely fills the texture (Wenzel state). It has an elastic modulus $E_{w} \simeq 1.4 \mathrm{GPa}$. The pillars, of height $e$, have a square cross-section of width $2 b$, and are organized on a square array of period $L$ (Figures $1 \mathrm{~b}$ and 1c). The solid surface fraction $\Phi$ of the solid-liquid interface at the top of the texture is thus $4 b^{2} / L^{2}$. Figure 1 c shows an example of the texture observed by scanning electron microscopy, with $\phi=0.3, e=85 \mu \mathrm{m}$ and $L=179$ $\mu \mathrm{m}$. The geometrical parameters of the textures used in this study are summarized in Table I, the precision on the measurement is $\pm 1 \mu \mathrm{m}$.

\section{The settling-sphere interferometer}

The container is inserted in an interferometric device (Figure 1a) in order that the top of the sphere reflects one of the laser beam, whereas the second beam is reflected by a fixed mirror at the bottom wall of the container. Interference fringes (circular rings) are formed and move according to the sphere displacement $([20])$. The recorded signal is the light intensity at the centre of the interference pattern. A signal variation from a maximum (bright fringe) to another maximum (bright fringe) is due to a displacement of the sphere equal to $\lambda / 2 n$, where $\lambda$ is the 
wavelength of the laser, and $n$ the index of refraction of the suspending fluid. Here, with a silicon oil $(n=1.404)$ and a He-Ne laser beam $(\lambda=632.8 \mathrm{~nm})$, we have $\lambda / 2 n \simeq 0.23 \mu \mathrm{m}$. The velocity $V(h)=-d h / d t$ of the sphere is related to the velocity of fringes displacement by multiplying $\lambda / 2 n$ by the signal frequency. After opto-electronic conversion by a photo-diode, the signal is recorded with an electronic oscilloscope at a sampling frequency of $25 \mathrm{MHz}$, transferred to a $\mathrm{PC}$ and processed by a Matlab code for detecting its extreme values as a function of time. The spatial resolution is therefore equal to $\simeq 0.23 \mu \mathrm{m}$. The maximal frequency of the signal that can be detected is of $0.5 \mathrm{MHz}$, yielding a time resolution of $0.002 \mathrm{~ms}$. The error on the measured frequency stems from the detection of the maxima. This is done by an automatic procedure and depends on the signal-to-noise ratio. It can be seen from Figure 2 that the signal-to-noise ratio deteriorates at small frequencies, because the low frequency limit of the oscilloscope is reached. The measured frequency has been averaged over 7.5 periods ( 75 points) so that the velocity is smoothed. This results in an error on the velocity smaller than $1 \%$. However, no averaging is applied in the vicinity of the contact, in order to capture the rapid variations of the velocity occuring in that region. The rest position $(h=0)$ of the sphere on top of the pillars (Figure $1 \mathrm{~b})$ is defined a posteriori when the period of the signal becomes very large, indicating a vanishing velocity, as depicted in Figure 2 . The resulting error on the contact position is of the order of the interfringe. The previous positions of the sphere are reconstructed by multiplying the velocity by the time interval.

It is important to note that when the sphere is sitting on the pillars, they are slightly compressed under the action of the apparent weight of the sphere. The static pillar deformation $\delta_{s}$ depends on the elastic modulus of the pillars, $E_{w}$, and on the geometrical parameters of the pillar array. Assuming an elastic longitudinal compression of the pillars yields $\delta_{s} / a=\left[4 e\left(\rho_{p}-\rho_{f}\right) g /\left(3 \Phi E_{w}\right)\right]^{1 / 2}$ ([9]). The values of $\delta_{s}$ are calculated to range between 0.8 and $3 \mu \mathrm{m}$ for the experiments presented here. The static deformation of the pillar will be neglected in the present analysis of the data. For comparison with the textured wall, the bottom glass surface of the container will provide a reference surface, and will be denoted in the following as the "smooth surface".

Figure 2 depicts the electronic signal obtained in the case of a sticking collision. Here a sphere of diameter $12.7 \mathrm{~mm}$ settles in the V1000 silicon oil toward the smooth surface. The sampling frequency is of $25 \mathrm{MHz}$. On the left of the top figure, the limit of the frequency detection is reached (frequencies larger than $0.5 \mathrm{MHz}$ ). As the sphere decelerates, the time modulation of the signal slows accordingly, until its ceases completely. The definitive arrest of the sphere is indicated by the vertical arrow. The bottom figure is a zoom on the signal, showing that a well defined frequency can be extracted from the dectection of the extrema of the signal. 

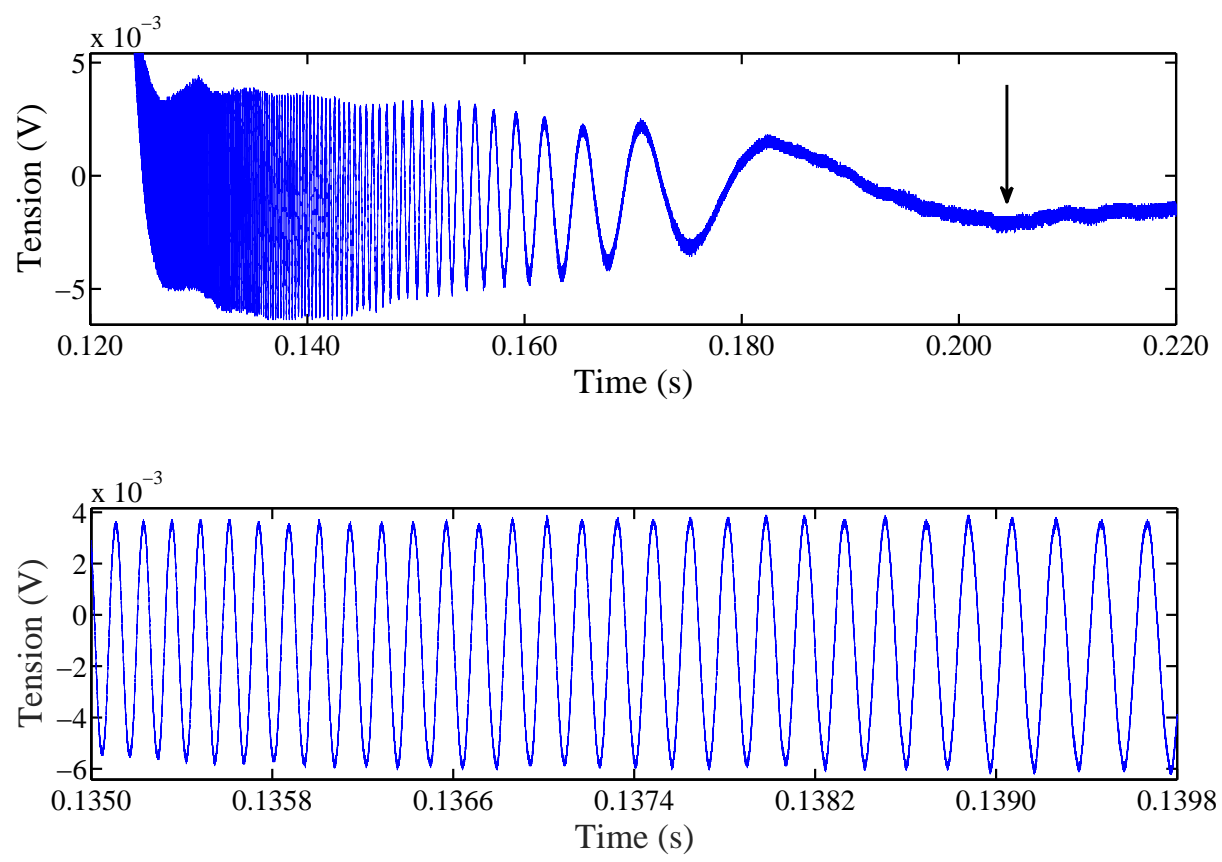

FIG. 2. Electronic signal recorded as a function of time during the settling of a steel sphere of diameter $2 a$ $=12.7 \mathrm{~mm}$ toward a smooth (glass) wall. The arrow indicates the arrest of the sphere on the surface.

\begin{tabular}{|c|c|c|c|c|c|c|c|c|c|c|c|c|c|c|c|c|c|c|c|}
\hline \multicolumn{5}{|c|}{$\Phi=0.05$} & \multicolumn{5}{|c|}{$\Phi=0.15$} & \multicolumn{5}{|c|}{$\Phi=0.3$} & \multicolumn{5}{|c|}{$\Phi=0.45$} \\
\hline$e$ & $2 b$ & $L$ & $s_{0}$ & $s_{e}$ & $e$ & $2 b$ & $L$ & $s_{0}$ & $s_{e}$ & $e$ & $2 b$ & $L$ & $s_{0}$ & $s_{e}$ & $e$ & $2 b$ & $L$ & $s_{0}$ & $s_{e}$ \\
\hline 9 & 56 & 240 & $8.6 \pm 0.3$ & $8.6 \pm 2$ & 9 & 49 & 141 & $7.3 \pm 0.3$ & $4.2 \pm 1$ & 9 & 96 & 176 & $5.1 \pm 0.3$ & $2.9 \pm 1$ & 9 & 96 & 142 & $4.2 \pm 0.3$ & $1.2 \pm 0.5$ \\
\hline 21 & 56 & 240 & $17 \pm 0.7$ & $12.7 \pm 2$ & 21 & 49 & 141 & $13.8 \pm 0.5$ & $7.7 \pm 1$ & 20 & 96 & 176 & $10.8 \pm 0.5$ & $5.9 \pm 1$ & 20 & 96 & 142 & $8.3 \pm 0.4$ & $0.6 \pm 0.5$ \\
\hline 57 & 56 & 240 & $43 \pm 2$ & $27.9 \pm 5$ & 60 & 57 & 145 & $31 \pm 1.5$ & $15.4 \pm 2$ & 57 & 102 & 177 & $18.7 \pm 0.6$ & $4.9 \pm 2$ & 60 & 95 & 148 & $13.8 \pm 0.6$ & $2.5 \pm 1$ \\
\hline 89 & 56 & 240 & $59 \pm 2$ & $32.8 \pm 5$ & 91 & 60 & 140 & $35.5 \pm 1.5$ & $15.2 \pm 3$ & 85 & 106 & 179 & $17.8 \pm 0.5$ & $5.9 \pm 2$ & 92 & 100 & 150 & $14.9 \pm 0.6$ & $1.4 \pm 1$ \\
\hline 130 & 56 & 240 & $71 \pm 3$ & $36.9 \pm 9$ & 117 & 55 & 148 & $35.5 \pm 1$ & $19.2 \pm 3$ & 121 & 100 & 179 & $26.5 \pm 3$ & $8.4 \pm 2$ & & & & & \\
\hline
\end{tabular}

TABLE I. Geometrical parameters of the arrays of micro-pillars (height $e$ and width $2 b$ of the pillars of square cross-section, and period $L$ of the array), and values of measured shift lengths $s_{e}$ and $s_{0}$ (all lengths are given in $\mu m)$.

\section{DRAG FORCE ON THE SPHERE NEAR A TEXTURED WALL}

In this section, the sphere settles in the most viscous oil so that the Reynolds and Stokes numbers are vanishingly small $\left(\simeq 10^{-5}\right)$. The settling sphere is subjected to a constant gravity force, which equilibrates the drag force on the sphere in this case of negligible sphere inertia. Figure 3 depicts the typical velocity-distance curves obtained for a sphere settling near a "smooth" wall and near two 
different arrays of pillars. Note that $h$ is the separation distance measured between the inferior pole of the sphere and the top of the asperities of the surface. Near a smooth wall, and within sufficiently small distances $(h / a<0.01)$, the velocity $V(h)$ is found to vary linearly with $h / a$, in agreement with the standard approximate lubrication drag force on the sphere $F_{D}(h)=6 \pi \eta a^{2} V(h) / h$. In practice, we obtain the value of the Stokes velocity $V_{S t}$ by adjusting to 1 the value of the slope when plotting the reduced velocity $V(h) / V_{S t}$ as a function of $h / a$. This procedure is indeed more precise than a calculation of $V_{S t}$, which would require to know the exact value of the viscosity of the oil at the temperature of the experiment. With increasing distances $(h / a>0.01)$, the curve deviates from the $X=Y$ plot, indicating that the lubrification approximation has to be corrected (see e.g. [21]).

For a sphere settling towards an array of micro-pillars, the velocity curves present two main differences with the one near a smooth wall. First, at a given distance $h$ from the top of the pillars, the sphere velocity is larger than it is at the same distance from a plain smooth wall. The hydrodynamic effect of a textured wall is therefore a drag reduction on the sphere in the near-wall region. A second effect of the texture is visible when approaching very close to the rest position of the sphere in the pillars (defined as $h=0$ ). Within a few micrometers, an abrupt deceleration occurs causing the complete arrest of the sphere. This deceleration is probably the signature of the solid contact and hence of the elastic deformation of the pillars ([9]). For the experiments shown in Figure 3, the contact starts at distances $h / a<0.001$, i.e at $h \cong 7 \mu \mathrm{m}$. The detailed analysis of this part of the velocity-distance curve is beyond the scope of the present paper. In the modelling section, we will ignore the pillar deformation and consider that the rest position $h=0$ correspond to the top of the (undeformed) pillars. This is equivalent to tolerate an error of $0.2 \%$ on the rest position $h=0$.

The amplitude of the velocity increase depends on the texture geometrical parameters ( [17]). In the example shown on Figure (3) of two textures with sensibly the same pillar height $(e=85$ $\mu \mathrm{m}$ and $e=89 \mu \mathrm{m})$, the velocity enhancement is more important for the smaller pillar fraction. Therefore, the drag reduction can be understood as a result of the fluid being able to escape between the pillars, thus decreasing the lubrication pressure. Alternatively, the top surface of the textured wall can be viewed as a composite surface with a patch of different hydrodynamic boundary conditions (no-slip over the pillars, effective slip over the liquid between the textures), from which an effective slip boundary condition can be obtained. The latter approach leads to the notion of an equivalent smooth plane (ESP), which provides an approximate but simple expression for the lubrication force on a sphere approaching a textured surface. We will use this notion in the 
following section.

Finally, the amplitude of the texture-induced velocity increase is spatially varying. The nearwall region can be divided into two sub-regions. In a so-called "far-field" region, the velocity curve near a textured wall is parallel to the one near a smooth wall, and appears merely as a shift to the left of this reference curve. In a so called "near-field" region, the velocity increase is more significant, with the velocity-distance relationship becoming non-linear just before the abrupt deceleration upon contact.

\section{A. The equivalent smooth plane model}

The notion of an ESP has been first introduced for modelling the drag on a millimetric sphere settling towards a corrugated wall [15]. It was also used for analysing experiments at a smaller scale : Atomic Force Microscope experiments in the case of a micrometric sphere with nano-rugosities [22] or in the case of a smooth sphere near a textured surface with micropillars [23], experiments using a Surface Force Apparatus in dynamic mode for a sphere near hydrophilic micro-textures [24]. As demonstrated in [15] and [18], the drag force on the sphere at a distance $h$ from the top of a periodic anisotropic surface (with corrugations or grooves) is the same as near a virtual smooth wall that would be situated at a distance $s_{e}$ down from the top of the corrugations (Figure 1b). This result is obtained by averaging the flow over a scale that is larger than a characteristic period of the corrugations and is thus valid only in the "far-field", that is for $h \gg L$. The length $s_{e}$ is denoted as the "effective shift length". The texture-modified drag force on the sphere for $L \ll h \ll a$ is thus given by :

$$
F_{D}^{*}(h) \simeq \frac{6 \pi \eta a^{2} V(h)}{h+s_{e}}
$$

Here, we recall that a shear flow along a wetted rough surface is equivalent to a shear flow along an effective smooth wall (see [15] and references therein). The effective slip length $b_{e}$ is defined as the distance, measured from the top of the roughness, at which the far-field flow, averaged over the roughness scale, extrapolates to zero. The averaged shear-stress $\left\langle\sigma_{w}\right\rangle$ at the slip plane (top of roughness) reads :

$$
\left\langle\sigma_{w}\right\rangle=\frac{\eta}{b_{e}}\left\langle u_{w}\right\rangle
$$

where $\left\langle u_{w}\right\rangle$ is the averaged slip velocity and $\eta$ the fluid viscosity. As proven in [15] and [18], Equation 1 yields the drag force on a sphere approaching an anisotropic textured wall on which 


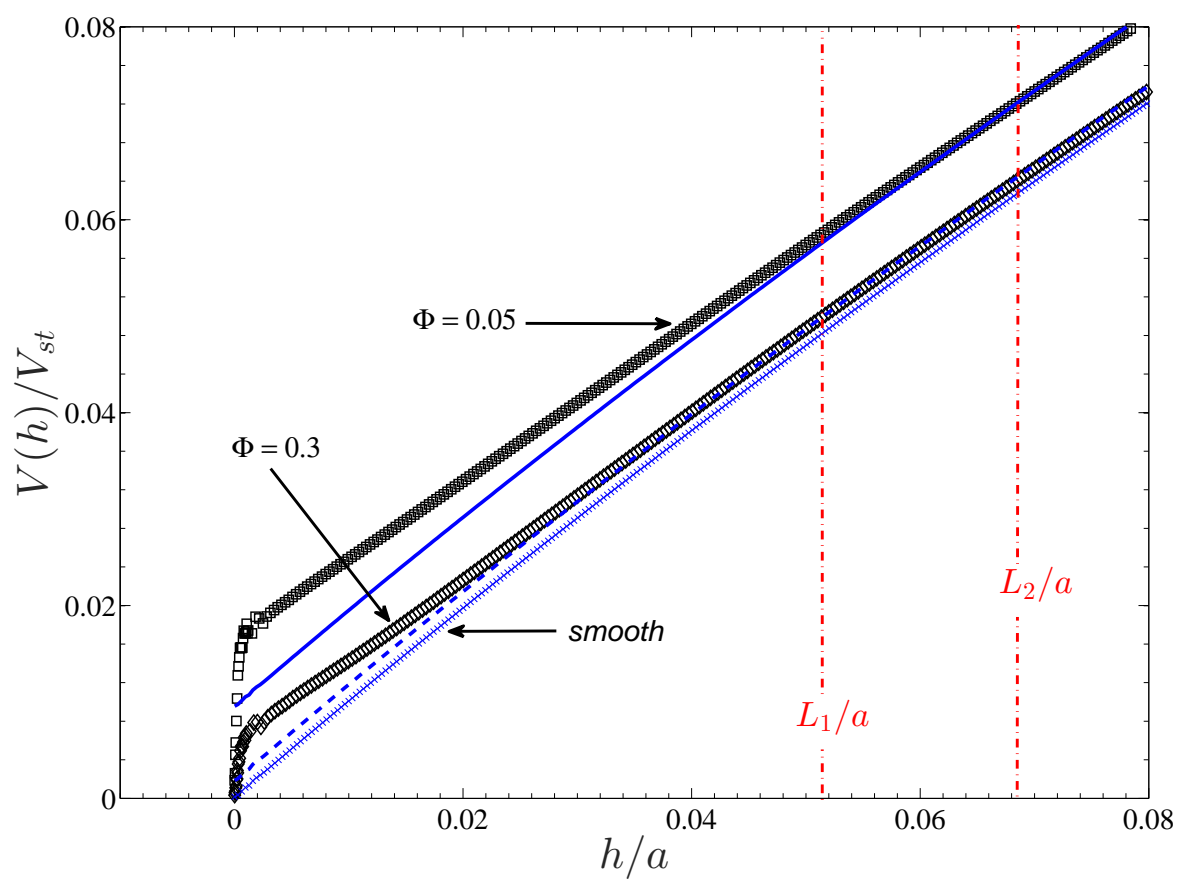

FIG. 3. Dimensionless velocity $V / V_{S t}$ measured as a function of dimensionless distance $h / a$ for a sphere of diameter $2 a=6.98 \mathrm{~mm}$ settling at small St and Re numbers towards different surfaces : smooth (glass) surface (crosses) ; array of micro-pillars with $\phi=0.3, e=85 \mu \mathrm{m}, L=L_{1}=179 \mu \mathrm{m}$ (diamonds) ; array of micro-pillars with $\phi=0.05, e=89 \mu \mathrm{m}, L=L_{2}=240 \mu \mathrm{m}$ (squares). Lines : experimental curves for a smooth surface shifted to the left along the horizontal axis with $s_{e}=5.9 \mu \mathrm{m}$ (dashed line) and $s_{e}=32.8$ $\mu \mathrm{m}$ (continuous line).

different effective slip lengths apply in two perpendicular directions. The shift length $s_{e}$ in Equation 1 is derived as the half-sum of longitudinal and transverse effective slip lengths $b_{/ /}$and $b_{\perp}$ for simple shear flows paralell and perpendicular to the corrugations $: s_{e} \simeq\left(b_{/ /}+b_{\perp}\right) / 2$. This theory was confirmed experimentally for periodic anisotropic textures in the Wenzel state $([15],[16])$ and in the Cassie state ([25]). It was anticipated in [16] that the same conclusion would hold for an isotropic texture with $b_{/ /}=b_{\perp}$. Hence, in principle, the shift length $s_{e}$ obtained from a measure of the drag on the sphere provides the effective slip length over the array of pillars. To our knowledge, only a few data [23] are available for the effective slip length over arrays of pillars in the Wenzel state.

Let us first check if the ESP model applies to our data. Using the texture-modified drag force $F_{D}^{*}(h)$ to balance the gravity forces on the sphere, $F_{g}=(4 / 3) \pi a^{3}\left(\rho_{p}-\rho_{f}\right) g$, yields the sphere velocity : 


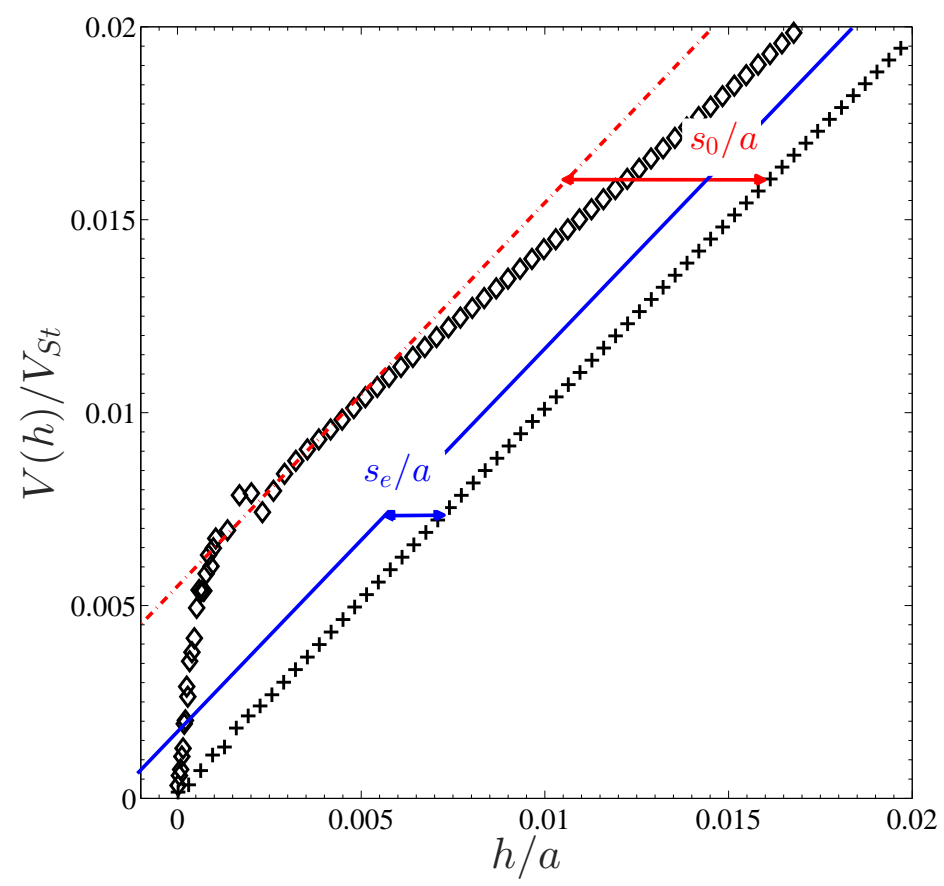

FIG. 4. Dimensionless velocity $V / V_{S t}$ measured as a function of dimensionless distance $h / a$ for a sphere of diameter $2 a=6.98 \mathrm{~mm}$ settling at small St and Re numbers towards different surfaces. Diamonds : array of pillars with $\phi=0.3, e=85 \mu \mathrm{m}, L=L_{1}=179 \mu \mathrm{m}$. Crosses : smooth (glass) surface. Continuous line : equation (2) with $s_{e}=5.9 \mu \mathrm{m}$. Dashed-dotted line : Equation (3) with $s_{e}$ replaced by $s_{0}=17.8 \mu \mathrm{m}$.

$$
\frac{V(h)}{V_{S t}}=\frac{h+s_{e}}{a}
$$

According to Equation 3, the velocity near a textured wall should thus be obtained by shifting to the left and along the horizontal axis the velocity-distance curve near a smooth wall. Figure 3 illustrates two typical cases. The far-field curves $(h / a \gg L / a)$ can indeed be reproduced by shifting the smooth-wall curve to the left, with shift lengths equal here to $s_{e} \simeq 5.9 \mu \mathrm{m}$ and $s_{e} \simeq 32.8 \mu \mathrm{m}$, respectively. Note that the far-field regions are distinct for the two cases, as the values of $L$ are different for the two textures under study $\left(L_{1}=179 \mu \mathrm{m}\right.$ and $\left.L_{2}=240 \mu \mathrm{m}\right)$. As expected from the theory, we observed that the shift procedure does not hold in the near-field, except however for the most diluted pillar array with the smallest pillar heights $(\Phi=0.05$ and $e=9 \mu \mathrm{m})$. The values of the shift length $s_{e}$ measured in the far-field for all the textures used in this studied are listed in Table I and displayed in Figure 5(a). We have checked that, using spheres of different diameters ranging between 6.98 and $12.7 \mathrm{~mm}$, settling near the same pillar array, sensibly similar values of $s_{e}$ are 
obtained. This indicates that the shift length is a characteristic only of the geometrical parameters of the pillar array, provided that $a \gg L$. The uncertainty on $s_{e}$ results from the variability due to the use of different sphere diameters, and from the precision of the shift procedure. For given geometrical parameters of the texture, this uncertainty does not exceed a few micrometers, as indicated in Table I. The shift length is an increasing function of pillar height $e$, and a decreasing function of pillar surface fraction $\Phi$. It can reach significant values compared to the values of the pillar height $e$. The most diluted pillar array $(\Phi=0.05)$ yields the largest value of $s_{e} \simeq 36.9 \mu \mathrm{m}$ for $e=130 \mu \mathrm{m}$.

At this point, it is useful to assess the values of the shift lengths $s_{e}$ obtained in the far-field region, by comparing them to predictions for effective slip lengths $L_{e}$ over wetted arrays of micropillars. This question is discussed in the next section.
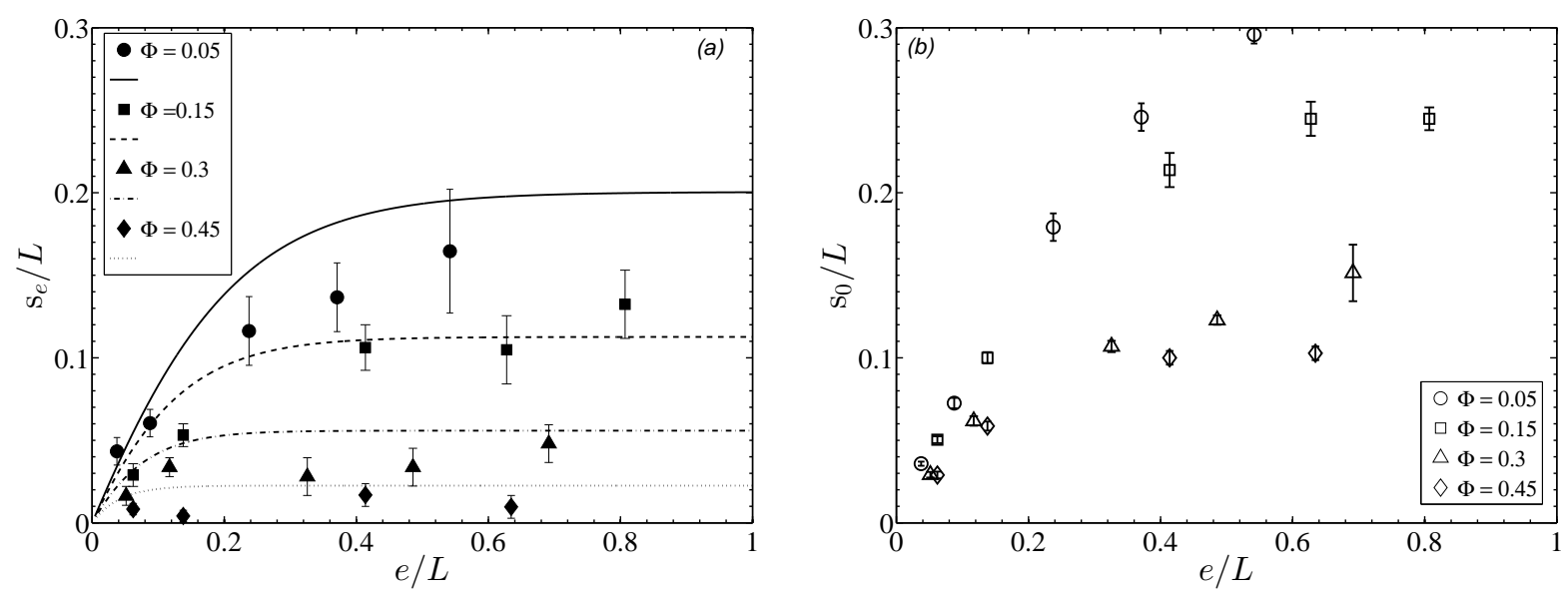

FIG. 5. Symbols : Values of dimensionless shift lengths $s_{e} / L$ (a) and $s_{0} / L$ (b) obtained experimentally in the far-field and in the near-field, respectively, as a function of dimensionless pillar height $e / L$, for textures of different pillar fractions $\Phi$ listed in Table I. (a) Lines : dimensionless slip length $L_{e} / L$ as predicted in [19] for $\eta / \eta_{g}=1$, with equations 4,5 and 6 .

\section{B. Effective slip length over a wetted array of micropillars}

Superhydrophobic surfaces, for which most of the interface between the texture and the liquid is a gas-liquid one (Cassie state), have been the subject of considerable interest because of their lowfriction properties [26]. Numerous modelling works have been devoted to the characterization of effective slippage arising on such surfaces. In contrast to conventional point of view, some of these theoretical approaches can be applied to situations where the ratio of the gas viscosity to the liquid 
viscosity is finite, thus encompassing the case of a wetted texture ([27]). We use here scaling laws proposed by Ybert et al. [19] for both unwetted and wetted surfaces in various geometries, including the case of wetted micro-pillars. Their heuristic modelling yields an approximate formula for the effective slip length over an array of micropillars as a function of generic surface characteristics, in particular the viscosity contrast between liquid and gas, the pillar's surface fraction and height. The physical grounds of this scaling are summarized below.

A first effective slip length $b_{\text {ideal }}$ is derived to account for the dissipation on top of the pillars. Similarly to the case of an idealized superhydrophobic surface, a flat surface is considered (neglecting the deformation of the gas-liquid interface), a shear-free flow between the pillars is assumed, and a no-slip boundary condition is imposed on top of the pillars. In the limit of low $\Phi$, a simple scaling argument coupling the spatial dependence of velocity components in Stokes flow predicts that $b_{\text {ideal }} / L \sim(0.325 / \sqrt{\Phi}-0.44)$. In the latter expression, the coefficients have been obtained numerically in [19] for $0.02 \leq \Phi \leq 0.3$. These coefficients were confirmed analytically within a few percent of variation for pillars of circular cross-section on a square lattice [28-30]. Note that these coefficients do not vary significantly when using pillars of square cross-section $[29,30]$.

For a wetted texture, the key point of the analysis is to consider the viscous dissipation on the pillar lateral walls, resulting in a finite effective slip over the gas phase. A second effective slip length is thus obtained, $\left((1-\Phi) b_{g}\right.$, where $b_{g}$ is the slip length at the gas-liquid interface taking into account the viscous dissipation. The latter is accounted for using an effective medium approach, and yields an effective slip length $b_{g}$ valid for finite pillar fractions $\Phi$ and moderate slip length $\left(b_{g} \ll L\right)[19]:$

$$
b_{g} \sim e \frac{\eta}{\eta_{g}} \frac{\tanh (\sqrt{\beta(\Phi)} e / L)}{\sqrt{\beta(\Phi)} e / L}
$$

where $\eta / \eta_{g}$ is the ratio of the viscosities of the liquid and the gas, respectively. The dimensionless function $\beta(\Phi)$ relates the drag force $f_{p}$ per unit length on a pillar in the array to the average velocity $U$ of the gas flow : $f_{p}=\beta(\Phi) \eta_{g} U$. It is an increasing function of the pillar surface fraction $\Phi$, and is available in the literature for arrays of cylindrical pillars only. We choose the expression given by Kuwabara ([31], [32]), valid for dilute pillar arrays $(\Phi \ll 1)$, and that we already used in [17] :

$$
\beta(\Phi) \sim \frac{4 \pi}{\ln \left(\frac{1}{\sqrt{\Phi}}\right)-0.75+\Phi-\frac{1}{4} \Phi^{2}}
$$

Finally, adding the frictions (Equation 2) stemming from the two sources of dissipation at the top of the pillars and at the lateral walls, yields the effective slip length $L_{e}$ as [19]:

$$
\frac{1}{L_{e}} \sim \frac{1}{(1-\Phi) b_{g}}+\frac{1}{b_{\text {ideal }}}
$$


Interestingly, the case of a hydrophilic slippage (Wenzel state) corresponds to $\eta=\eta_{g}$. We thus calculate the effective slip length $L_{e}$ from equations 4,5 , and 6 with $\eta / \eta_{g}=1$ and different values of the surface fraction $\Phi$ of the pillars. The resulting predictions for $L_{e} / L$ are shown on Figure 5(a) together with the measured values of dimensionless shift lengths $s_{e} / L$. Clearly, the evolution of the measured shift lengths with $e / L$ and $\Phi$ follows the trends predicted by the slip length predictions. It is interesting to discuss the asymptotic behaviors predicted by the scaling laws at a given pillar fraction $\Phi$. In the limit of small pillar heights $(e / L \ll 1)$, equation 4 reduces to $b_{g} \sim e$. At small pillar fraction $(\Phi \ll 1), b_{\text {ideal }}^{-1}$ is negligible compared to $\left((1-\Phi) b_{g}\right)^{-1}$, and equation 6 yields :

$$
\frac{L_{e}}{L} \simeq \frac{e}{L}(1-\Phi)
$$

indicating a linear increase of $L_{e}$ with $e$, with a slope that increases with decreasing pillar fraction $\Phi$. In the other limit of large pillar heights $(e / L \gg 1)$, equation 4 reduces to $b_{g} \sim L / \sqrt{\beta(\Phi)}$. Here, the resulting value of $L_{e} / L$ is independent of the pillar height $e$ :

$$
\frac{L_{e}}{L} \simeq \frac{1}{\frac{\sqrt{\beta(\Phi)}}{(1-\Phi)}+\frac{1}{0.325 / \sqrt{\Phi}-0.44}}
$$

It is visible from Figure 5(a) that the experimental shift lengths $s_{e} / L$ follow approximately these asymptotic behaviors. The saturation of $L_{e} / L$ predicted by the model at large pillar heights is indeed observed experimentally on $s_{e} / L$ for the three largest pillar fractions studied here, with a quantitative agreement even obtained for some of the data, particularly for $\Phi=0.15$. However, for the most dilute pillar arrays $(\Phi=0.05)$, the predicted plateau value of $L_{e} / L \simeq 0.19$ is not reached experimentally. Conversely, for the same solid fraction $\Phi=0.05$ but at small pillar heights, the linear evolution of $s_{e}$ with $e$ is in very good agreement with the model predictions. These comparisons show that effective shift lengths measured via the ESP from the drag on a sphere approaching a square array of micropillars in the Wenzel state are strongly related to effective slip lengths over these arrays.

\section{Modelling the near-field}

In the near-field region, $h \ll L$, an alternative description is needed for the drag reduction on the sphere. The difficulty stems from the fact that the velocity-distance curve is non-linear in that region. In a previous work, we shown that this non-linearity could be handled by the lubrication flow of two adjacent fluid layers, the bottom layer fluid having a viscosity that reflects the viscous

dissipation through the array of pillars [17]. Here, we have chosen for simplicity to keep the same 
convenient form of the drag with a separation-independent shift length, as given by Equation (1), but with a near-field shift length denoted $s_{0}$. The latter could be obtained analytically in the asymptotic limit of $h \ll L$, but this is beyond the scope of the present work. We thus apply a fitting procedure to the sphere velocity by Equation (3) with $s_{e}$ replaced by $s_{0}$. As shown on Figure 4 , the value of $s_{0}$ is chosen in order that the fitting velocity is tangent to the maximal value of the measured velocity in the near-field. It is clear from Figure 4 that this one-parameter model is very approximate. However, it is representative of the near-field velocity as it describes correctly the maximum velocity enhancement in the near-field. The values of $s_{0}$ obtained by this same procedure as shown in Figure 4 are listed in Table I and plotted in Figure 5(b). Essentially, the values of $s_{0}$ follow the same trend as $s_{e}: s_{0}$ is an increasing function of pillar height $e$ (with a saturation beyond a certain value of pillar height) and increases with decreasing pillar fraction $\Phi$. However, for a given pillar array, $s_{0}$ values are generally larger than the value of $s_{e}$ obtained in the far field, reflecting the fact that the velocity enhancement is more pronounced in the near-field than in the far-field. Only for the most diluted pillar array with the smallest pillar heights $(\Phi=$ 0.05 and $e=9 \mu \mathrm{m}$ ), the values of $s_{0}$ and $s_{e}$ are found nearly identical. The near-field description of the drag force with $s_{0}$ will be used in the modelling of section IV.

\section{STICKING COLLISION WITH A TEXTURED WALL}

In this section, we investigate the motion of the sphere near the same textured walls as in the previous section, but the sphere inertia is here non-negligible. We use spheres of different diameters and densities in the less viscous oil 47V1000, so that the Stokes numbers are in the range between $2.5 \leq S t \leq 6.8$ (see Table II). For that range of particle Stokes numbers, care was taken to select textures with geometrical parameters such that no appreciable rebound was detected subsequent to impact with the wall.

\section{A. Analysis of velocity-distance curves}

Figure 6(a) illustrates the effect of the Stokes number on the sphere motion near a given textured wall, here a micro-pillar array of pillar fraction $\Phi=0.05$, and pillar height $e=21 \mu \mathrm{m}$. The sphere diameter is fixed, and the Stokes number has been varied using two different oil viscosities. The velocity $V(h)$ is made dimensionless by a characteristic velocity $V^{*}$ that depends on the Stokes number value. In the case of $S t \ll 1$, this characteristic velocity $V^{*}$ is the Stokes velocity $V_{S t} \simeq$ 


\begin{tabular}{|c|c|c|c|c|c|c|c|c|}
\hline Materials & $2 \mathrm{a}(\mathrm{mm})$ & $V_{S t}(\mathrm{~mm} / \mathrm{s})$ & $V_{T}(\mathrm{~mm} / \mathrm{s})$ & $\mathrm{Re}$ & $\mathrm{St}$ & $V_{0}(\mathrm{~mm} / \mathrm{s})$ & $\left(V_{0}-V_{S t}\right) / V_{0}(\%)$ & $F r_{0}$ \\
\hline Steel & 10.5 & 420 & 271 & 2.9 & 2.5 & 427 & 1.7 & 4.3 \\
- & 12.7 & 612 & 337 & 4.3 & 3.8 & 643 & 5.1 & 8.11 \\
- & 14 & 747 & 373 & 5.3 & 4.7 & 828 & 10.8 & 12.2 \\
- & 15 & 857 & 399 & 6.0 & 5.4 & 950 & 10.9 & 15.0 \\
- & 15.9 & 960 & 421 & 6.7 & 6.8 & 1069 & 11.4 & 17.7 \\
Carbid tungsten & 8 & 523 & 341 & 2.8 & 4.9 & 612 & 17.2 & 10.55 \\
\hline
\end{tabular}

TABLE II. Data for spheres of different materials and diameters settling in 47V1000 silicon oil.The Stokes velocity, $V_{S t}$, and the terminal velocity, $V_{T}$, are the velocities of the sphere calculated in the corresponding unbounded fluid, using a Stokes drag force, and a drag corrected for inertia effects, respectively. Re and $S t$ are based on the terminal velocity $V_{T}$. $V_{0}$ is the characteristic velocity measured in the linear part of the velocity distance-curves, and $F r_{0}$ is a Froude number based on this velocity $V_{0}$ (Equation 12).

$612 \mathrm{~mm} / \mathrm{s}$. Thus, $V(h) / V^{*}$ shows a linear variation with dimensionless distance $h / a$, before a rapid deceleration, as discussed in the previous section.

In the case of finite Stokes number, here $S t \simeq 3.8$, the velocity curve exhibits an additional regime before collapsing on the two previous (linear followed by a rapid deceleration) regimes obtained at $S t \simeq 0$. This additional regime is strongly non-linear, and corresponds to the balance of sphere inertia and lubrication drag force. It therefore holds as long as the sphere inertia is much larger than gravity forces. At a certain distance, however, the deceleration of the sphere upon the action of the lubrication force is such that its inertia becomes negligible compared to gravity forces. Thus, a linear regime is recovered, corresponding to the balance of gravity and lubrication drag forces. Finally, the last regime corresponds to the rapid deceleration due to the penetration of the sphere into the pillars, eventually leading to a vanishing velocity. In that case, the value of the characteristic velocity $V^{*}$ obtained in the linear regime is $\simeq 643 \mathrm{~mm} / \mathrm{s}$, larger by $\simeq 5 \%$ than the Stokes velocity. In the following, we will denote as $V_{0}$ this characteristic velocity $V^{*}$ obtained at finite Stokes number in the linear part of the curve. The physical origin of this characteristic velocity $V_{0}$ is not clear to us. We measure a value of $V_{0}$ that is always larger than $V_{S t}$. The discrepancy increases with increasing $R e$ for the steel spheres, but this trend has not be verified with the carbid tungsten sphere. Our hypothesis is that the value of $V_{0}$ may reflect the effect on the lubrication drag of the non-steady fluid motion at finite Renolds number [14].

Interestingly, when using different surfaces with the same sphere and same fluid, as depicted in Figure 6(b), we observe that linear regimes have essentially the same slope. Hence, it is tempting for 
simplification purposes to describe them by the same velocity $V_{0}$, independently on the geometrical parameters of the surface, either smooth or textured. In the following we will consider, without loss of generality, that $V_{0}$ is a characteristic of the particle and fluid only, and hence, of a given particle Stokes number. As a consequence, we measured the value of $V_{0}$ using the velocity curve near a smooth wall, for which the linear regime has the largest spatial extension. The values of $V_{0}$ obtained by this procedure are listed in Table II for the particles used in this study.
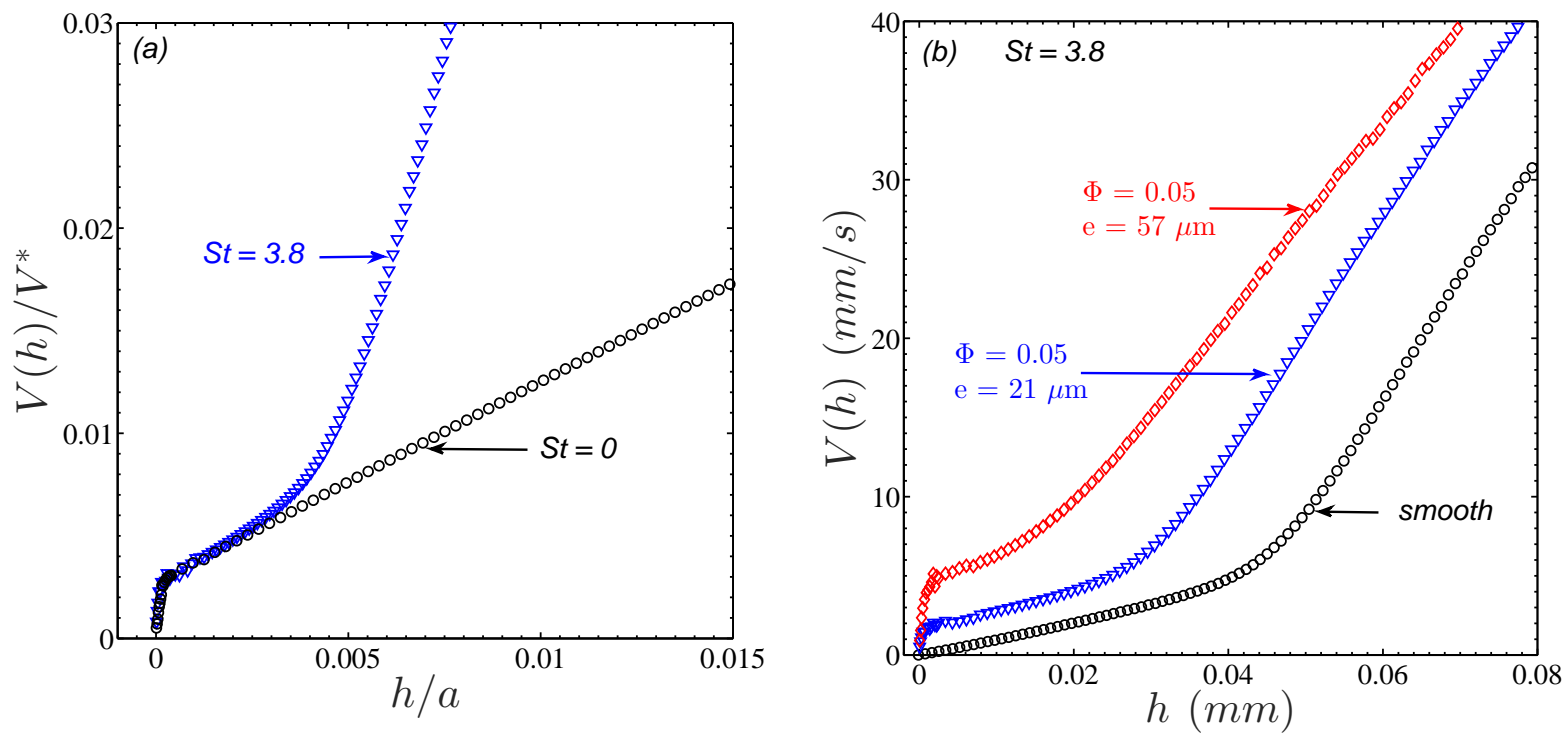

FIG. 6. (a) Dimensionless velocity $V / V^{*}$ as a function of dimensionless sphere-to-wall distance $h / a$, for a steel sphere of diameter $2 a=12.7 \mathrm{~mm}$ settling near the same array of micro-pillars $(\phi=0.05, e=21 \mu \mathrm{m})$ in : (circles) $47 \mathrm{~V} 100000$ silicon oil at $S t \simeq 0$ with $V^{*}=V_{S t}=612 \mathrm{~mm} / \mathrm{s}$, (triangles) $47 \mathrm{~V} 1000$ silicon oil at $S t=3.8$ with $V^{*}=V_{0}=643 \mathrm{~mm} / \mathrm{s}$. (b) Velocity-distance curve for a steel sphere of diameter $2 a=12.7$ $\mathrm{mm}$ settling in $47 \mathrm{~V} 1000$ silicon oil at $\mathrm{St}=3.8$ near a smooth surface and arrays of micro-pillars.

The Figures 7, 8, and 9 show the variety of dynamics obtained experimentally by playing with different texture geometries, sphere diameters and sphere densities. The velocity-distance curves are plotted with the velocity made dimensionless by $V_{0}$, the characteristic velocity measured in the linear regime for a given Stokes number. Indeed, the linear regime is always found to be parallel to the $X=Y$ line, which validates our hypothesis on $V_{0}$. The Figure 7 (a) illustrates the effect of increasing the particle Stokes number for a given textured wall : the non-linear regime is extended to the detriment of the linear regime, as a consequence of the sphere entering the near-wall region with a higher velocity, the higher the Stokes number. In this example, a very short linear regime is still detectable at the largest Stokes number values used here $(S t=5.4)$. The Figures $7(\mathrm{~b})$ and 

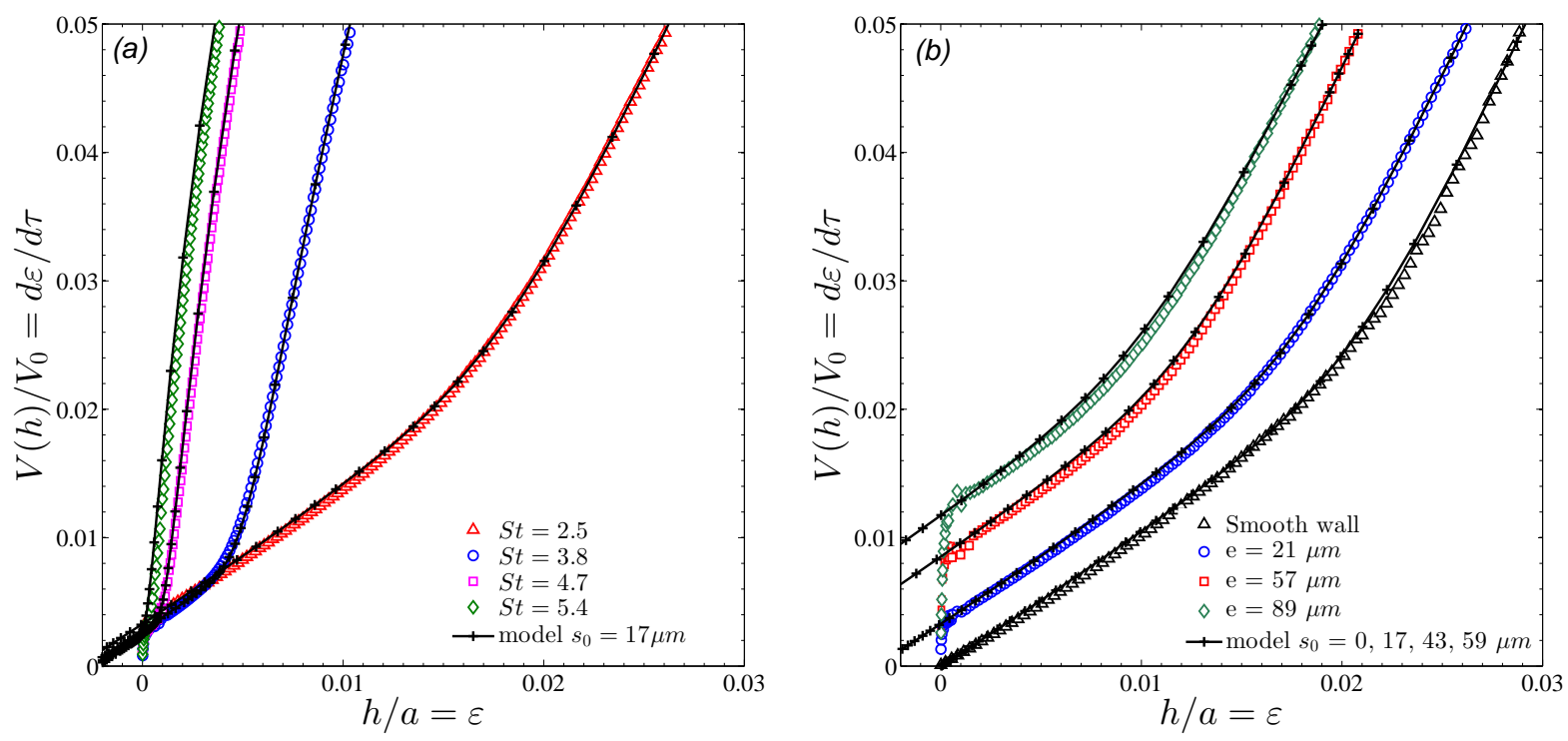

FIG. 7. Dimensionless velocity $V / V_{0}=d \epsilon / d \tau$ as a function of dimensionless sphere-to-wall distance, $h / a=\varepsilon$, of steel spheres (a) of different diameters near a square array of micro-pillars of pillar height $e=21 \mu \mathrm{m}$ and pillar concentrations $\Phi=0.05$. Symbols : experiments. Solid lines : solution of Equation 11 with $s_{0}=17$ $\mu \mathrm{m}$. (b) of same diameter $(S t=2.5)$ near square arrays of micro-pillars of pillar concentration $\Phi=0.05$ and different pillar heights. Symbols : experiments. Solid lines : solution of Equation 11 with $s_{0}=0,17$, 43 and $59 \mu \mathrm{m}$.

8(b) show the effect of increasing the pillar height at a given Stokes number : the velocity curves are shifted, yielding higher values of velocity at a given distance $h$, the higher the pillar height. Interestingly, the spatial extension of the linear regime is here reduced by increasing the pillar height, as a consequence of the sphere velocity being locally increased by the textured wall. The Figure 8(a) shows that an analogous effect is obtained by decreasing the pillar fraction at a given Stokes number and a given pillar height. Data obtained with a tungsten carbide sphere in Figure 9 follow the same trends as for the steel spheres.

\section{B. Modelling}

This section aims to describe the sphere dynamics before the contact, focusing on the description of the first two regimes. We extend the semi-empirical model previouly used for the sticking collision on a smooth surface ([14]) by using, in the equation of motion of the sphere, a lubrication drag force that is modified by the presence of the texture. In order to describe experimental data situated in the region $h \ll L$, we use the near-field shift lengths $s_{0}$ measured in section III, thus assuming that 
shift-lengths are not modified at these finite Reynolds number values. We use an ad-hoc correction factor for the drag force, equal to the ratio $V_{S t} / V_{0}$, in order to describe the linear regime. The latter linear regime differs from the actual lubrication regime obtained at $S t \simeq 0$, as the characteristic velocity is found here to be $V_{0}$ and not $V_{S t}$. Finally, the modified drag force reads :

$$
F_{D}^{* *}(h)=\frac{V_{S t}}{V_{0}} \frac{6 \pi \eta a^{2} V(h)}{h+s_{0}}
$$

Inertial effect of the fluid are taken into account by including the contribution of added mass to the forces on the sphere. The added mass coefficient $C_{M}$ for a sphere near a wall is a function of the distance to the wall, and has been shown to be the same at $R e=O(1)$ as in a potential flow [10] : it slightly increases from the value $C_{M}=0.5$ far from the wall, to a value of $C_{M} \simeq$ 0.563 at the wall $(h=0)$. Our experiments are situated in the range $h / a<0.03$, so that we choose a constant value $C_{M} \simeq 0.563$ corresponding to the limit of vanishing $h$. Note that the added mass contribution to sphere inertia is very small in the case of a tungsten carbide sphere, but is more significant for steel spheres. The question of history force may be more complicated : to our knowledge, an analytical expression for the history force including a wall effect is unknown for $R e=O(1)$. Thus, the equation of motion of the sphere is simply written as :

$$
\frac{4}{3} \pi a^{3}\left(\rho_{p}+C_{M} \rho_{f}\right) \frac{d V(h)}{d t}=-F_{D}^{* *}(h)+\frac{4}{3} \pi a^{3}\left(\rho_{p}-\rho_{f}\right) g
$$

with $V(h)>0$ corresponding to the approach to the wall. Defining a dimensionless time $\tau=$ $t V_{0} / a$ and a dimensionless distance $\varepsilon=h / a$, and dividing Equation 10 by the gravity forces, a dimensionless form of the equation of motion is obtained as :

$$
-F r_{0} \frac{d^{2} \varepsilon}{d \tau^{2}}=\left[\frac{1}{\varepsilon+s_{0} / a}\right] \frac{d \varepsilon}{d \tau}+1
$$

where $F r_{0}$ is a Froude number modified by the added mass and based on the velocity $V_{0}$ :

$$
F r_{0}=\frac{\left(\rho_{p}+C_{M} \rho_{f}\right) V_{0}^{2}}{\left(\rho_{p}-\rho_{f}\right) g a}
$$

$F r_{0}$ is the ratio of the sphere kinetic energy to its apparent gravitational energy. Note that this Froude number (and not the Stokes number) appears in Equation 11 as a result of dividing Equation 10 by the gravity forces (and not by the viscous forces).

In summary, Equation 11 indicates that the dynamics of the sphere approaching a given texture at a given Stokes number is driven by two parameters : a Froude number $F r_{0}$ based on the velocity 
$V_{0}$ that is a characteristic of the sphere and the fluid, and a dimensionless near-field shift length $s_{0} / a$ that is a characteristic of the texture relative to sphere radius. These parameters are empirically known as follows : $V_{0}$ is measured in the linear regime obtained at the same Stokes number near a smooth wall, and $s_{0}$ is measured in settling experiments towards the same texture at $S t \simeq 0$.
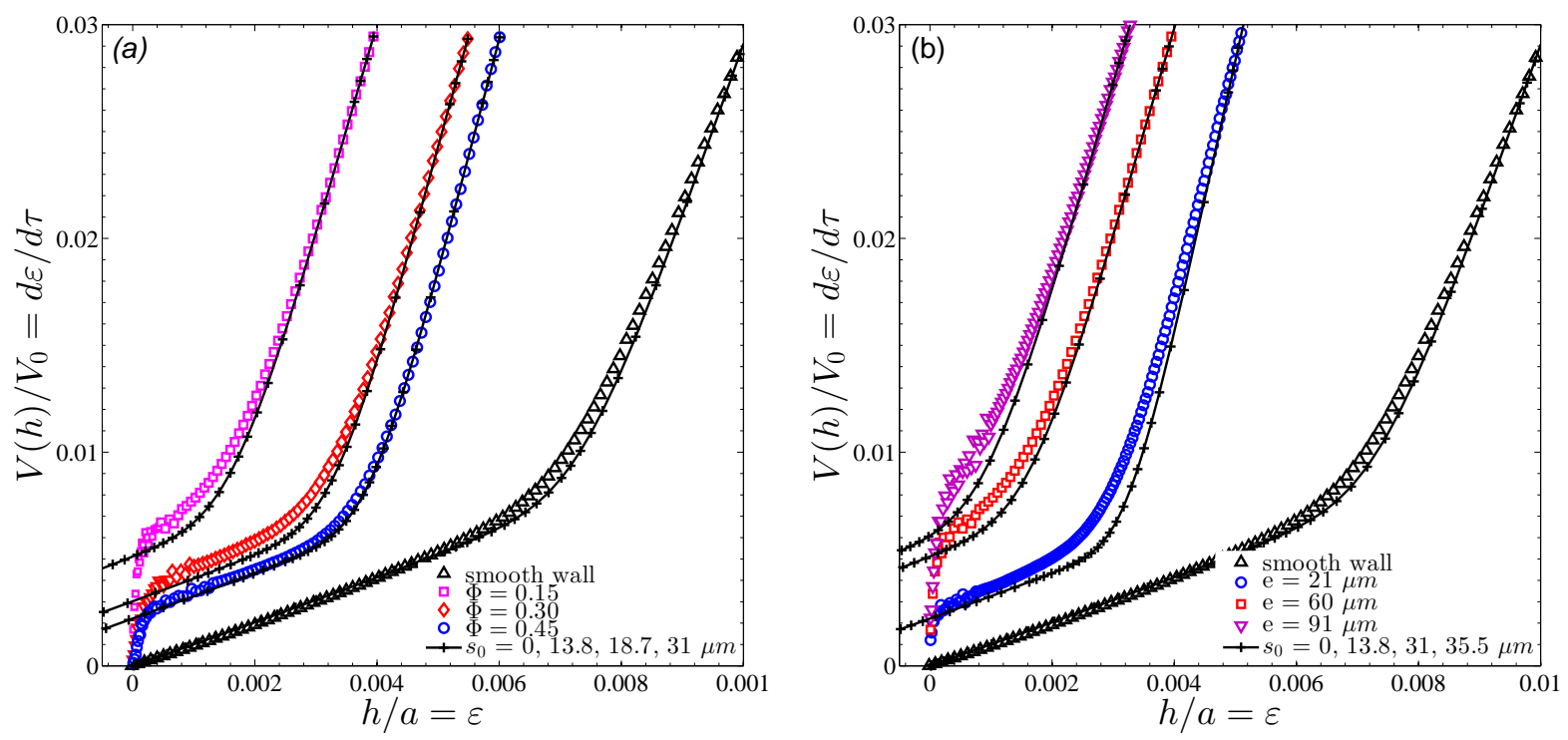

FIG. 8. Dimensionless velocity $V / V_{0}=d \epsilon / d \tau$ as a function of dimensionless sphere-to-wall distance, $h / a=\varepsilon$, of a steel sphere of diameter $2 a=12.7 \mathrm{~mm}(S t=3.8)$, near different square arrays of micro-pillars. (a) : same pillar height $e=60 \mu \mathrm{m}$ but different pillar concentrations $\Phi$. Symbols : experiments. Solid lines : solution of Equation 11 with values of $s_{0}$ (in $\mu \mathrm{m}$ ) : 31, 18.7, 13.8, 0. (b) : same pillar concentration $\Phi=$ 0.15 but different pillar heights. Symbols : experiments. Solid lines : solution of Equation 11 with values of $s_{0}($ in $\mu \mathrm{m}): 35.5,31,13.8,0$.

Equation 11 is solved numerically choosing as initial conditions some experimental values of $(\varepsilon$, $d \varepsilon / d \tau)$ in the near-wall region. The program is stopped at sufficiently long times for the values of $d \varepsilon / d t$ to be close to zero. Concerning the sphere dynamics near a textured wall, the velocities obtained at negative values of the distance $h$ are unphysical, as the contact interaction between sphere and pillars is ignored in the model. In the following, the $h<0$ part of the numerical curves will be displayed for convenience, however the discussion of the numerical results will be restricted to the region prior to the contact of the sphere with the pillars.

The results of the modelling are plotted in Figures 7, 8 and 9 as continuous lines. In the case of a smooth wall, we obtain a perfect agreement between the model (using $s_{0}=0$ ) and the experiments, as already observed in [14]. This agreement validates the simplifying assumptions of our model, 
i.e. the neglect of a history force, and the use of a correction factor $V_{S t} / V_{0}$. In the case of textured walls, the consequence of using a shift length $s_{0}$ in the drag force is, as expected, to enhance the velocity compared to the case of a smooth wall. Strikingly, the model yields excellent results by reproducing the experiments obtained for the smallest pillar surface fraction $\Phi=0.05$, as depicted in Figure 7. This indicates that the effect of a given texture on the drag force at $S t \simeq O(1)$ is here accurately described by the shift lengths $s_{0}$ measured in experiments at $S t \simeq 0$ and listed in Table I. For the other textures with larger pillar fractions, the quantitative agreement between model and experiments is however less remarkable, particularly in the linear regime, as can be seen in Figure 8 and Figure 9. In practice, the agreement between model and experiments could be improved by using $s_{0}$ as an adjustable parameter in Equation 11. For instance, the values of $s_{0}$ obtained by fitting the model to the experimental data in Figure 9 are 18, 12 and $6 \mu \mathrm{m}$, instead of the values $17.8,13.8$ and $8.3 \mu \mathrm{m}$ taken respectively from Table I, yielding a maximum relative difference of $13 \%$. Despite its limitations, we believe that Equation 11 remains a convenient tool to understand and predict how the texture geometrical parameters influence the sphere dynamics in the near wall region at $S t \simeq O(1)$.

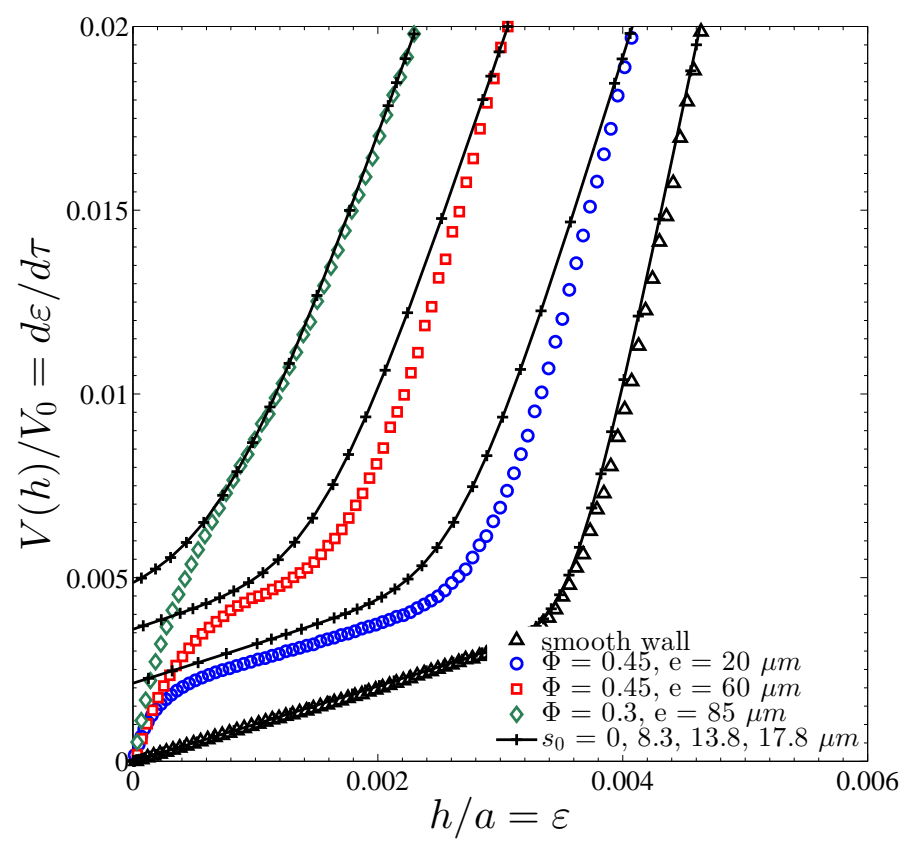

FIG. 9. Dimensionless velocity $V / V_{0}=d \epsilon / d \tau$ as a function of dimensionless sphere-to-wall distance, $h / a=\varepsilon$, of a carbide tungstene sphere of diameter $2 a=8 \mathrm{~mm}$, settling toward different arrays of micro-pillars at $S t$ $=4.9$. Symbols : experiments. Solid lines : solution of Equation (11) with values of $s_{0}$ (in $\left.\mu \mathrm{m}\right): 17.8,13.8$, $8.3,0$. 


\section{Towards the bouncing collision}

The bouncing of the sphere will occur as a consequence of the disappearance of the linear regime, leading to a finite sphere velocity at contact. In the non-linear regime, equation 10 reduces to the balance between sphere inertia and lubrication drag force. Integrating the two terms leads to a velocity varying as :

$$
\frac{V(h)}{V_{T}}=1+\frac{1}{S t} \frac{V_{S t}}{V_{0}} \ln \left[\frac{h+s_{0}}{a+s_{0}}\right]
$$

We have used as boundary condition $V(h=a)=V_{T}[11]$, considering that the lubrication drag overwhelms the Stokes drag at a distance from the wall of the order of the sphere radius. A necessary condition for rebound to occur is that the contact velocity is non-zero. Writing $V(h=0)>0$ yields thus a lower bound for the critical Stokes number, $S t_{c}>S t_{m i n}$, with :

$$
S t_{\text {min }}=\frac{V_{S t}}{V_{0}} \ln \left[\frac{a+s_{0}}{s_{0}}\right] \simeq \frac{V_{S t}}{V_{0}} \ln \left[\frac{a}{s_{0}}\right]
$$

This criteria is similar to the one established for surfaces with a micro-roughness ([4], [11]). Here, instead of a characteristic roughness height, we use the texture parameter $s_{0}$ that accounts for the effective modification of the drag force by the texture. Hence, the model predicts that the higher is the value of $s_{0}$, the smaller is the value of minimum critical Stokes number for bouncing, consistently with the experimental observations $([9])$. With the values of $s_{0}$ obtained in this work, that are of the order of $\simeq 10 \mu \mathrm{m}$, and considering that $V_{S t} \simeq V_{0}$, the minimum value of Stokes number for bouncing is predicted by Equation 14 to be $S t_{\min } \simeq 6.7$ for the largest sphere of diameter $2 a=$ $15.9 \mathrm{~mm}$. For the same sphere diameter, Equation 14 yields a minimum Stokes number of $S t_{\min } \simeq$ 11.3 for bouncing on a surface of micro-roughness $s_{0} \simeq 0.1 \mu \mathrm{m}$. These predicted values of $S t_{\min }$ are consistent with experiments reported in the literature ([7], [8], [9]). It is important to note however that a vanishing linear regime is a necessary but non-sufficient condition : as illustrated in Figure 9 for the case $\Phi=0.3$, the kinetik energy may be entirely dissipated in the contact process and no rebound is observed.

\section{CONCLUSIONS AND PERSPECTIVES}

Velocity measurements of a sphere settling towards a textured wall have been performed in the region close to contact with the wall. High frequency interferometry has been used to resolve in time and space the sphere dynamics in that region. The main effect of the wall texture, here arrays of micro-pillars of square cross-section, is to enhance the sphere velocity, measured from the top 
of the pillars, compared to the case of the smooth wall. This effect is significant in the region of interest.

In the case of negligible sphere inertia, which in our experiments corresponds also to the case of negligible fluid inertia, we have shown that this velocity enhancement can be described in the far-field using the notion of an equivalent smooth plane that is shifted down from the top of the textures. We have extracted shift-lengths for each texture geometry, and their values compare reasonably well to scaling laws for effective slip-lengths over micro-pillars in the Wenzel state.

In the case of non-negligible sphere inertia, we have characterized different dynamics of sticking collision of the sphere with a textured wall. By sticking collision, we mean that the sphere collides the top of the texture with a finite but sufficiently small velocity so that all its kinetic energy is dissipated during its penetration into the pillars, and thus no rebound occurs. The experiments reveal three distinct regimes : a non-linear regime, a lubrication-like linear regime, and a final deceleration corresponding to the sphere penetration into the pillars. Limiting our analysis to the first two regimes, we have shown that they result from the competition between sphere inertia and lubrication drag force in the near-field region. An interesting effect of the texture is, by enhancing locally the sphere velocity, to modify spatially the relative importance of these forces for a given particle. As a result, for a sphere of given diameter and density, the contact velocity when hitting the top of the texture is strongly influenced by the geometrical parameters of the texture. The observed sphere dynamics can be well accounted for using a shift-length-modified drag force in the equation of motion. In particular, by enabling the prediction of the sphere velocity at contact, our modelling is a first step towards the description of the dynamics of contact and of subsequent microrebounds. During the contact, the elastic resistance of the pillars to the compression under the action of sphere inertia and gravity forces has to be taken into account [9]. An analysis combining the elastic deformation of the pillars and the viscous dissipation by the shift-length-modified drag force, is under progress.

\section{ACKNOWLEDGMENTS}

We thank Philippe Gondret, Ory Schnitzer and Lydéric Bocquet for fruitful discussions.

[1] R. H. Davis, "Elastohydrodynamic collision of particles," Physico-Chem Hydrodyn. 9, 41-52 (1987). 
[2] J. R. Smart and D. T. Leighton, "Measurement of the hydrodynamic surface roughness of noncolloidal spheres," Phys. Fluid A 1, 52-60 (1989).

[3] R. H. Davis, J. M. Seyrassol, and E. J. Hinch, "The elastohydrodynamic collision of two spheres," J. Fluid Mech 163, 479-497 (1986).

[4] G. Barnocky and R. H. Davis, "Elastohydrodynamic collision and rebound of spheres : experimental verification," Phys. Fluids 31(6), 1324-1329 (1988).

[5] R. H. Davis, D. A. Rager, and B. T. Good, "Elastohydrodynamic rebound of spheres from coated surfaces," J. Fluid Mech 468, 107-119 (2002).

[6] P. Gondret, E. Hallouin, M. Lance, and L. Petit, "Experiments on the motion of a solid sphere toward a wall : from viscous dissipation to elastohydrodynamic bouncing," Phys. Fluids 11(9), 2803 (1999).

[7] G. G. Joseph, R. Zenit, M. L. Hunt, and A. M. Rosenwinkel, "Particle-wall collisions in a viscous fluid," J. Fluid Mech. 433, 329-346 (2001).

[8] P. Gondret, M. Lance, and L. Petit, "Bouncing motion of spherical particles in fluids," Phys. Fluids 14(2), 643 (2002).

[9] T. Chastel, A. Mongruel, and P. Gondret, "Texture-driven elastohydrodynamic bouncing," J. Fluid Mech. 805, 577-590 (2016).

[10] A. M. Ardekani and R. H. Rangel, "Numerical investigation of particle-particle and particle-wall collision in a viscous fluid," J. Fluid Mech 596, 437-446 (2008).

[11] E. Izard, T. Bonometti, and L. Lacaze, "Modelling the dynamics of a sphere approaching and bouncing on a wall in a viscous fluid," J. Fluid Mech. 747, 422-446 (2014).

[12] F.L. Yang and M. L. Hunt, "A mixed contact model for an immersed collision between two solid surfaces," Phil. Trans. R. Soc. Lond. A 366, 2205-2218 (2008).

[13] J.O. Marston, W. Yong, and S. Thoroddsen, "Direct verification of the lubrication force on a sphere travelling through a viscous film upon approach to a solid wall," J. Fluid Mech. 655, 515-526 (2010).

[14] A. Mongruel, C. Lamriben, S. Yahiaoui, and F. Feuillebois, "The approach of a sphere to a wall at finite Reynolds number," J. Fluid Mech. 661, 229-238 (2010).

[15] N. Lecoq, R. Anthore, B. Cichocki, P. Szymczak, and F. Feuillebois, "Drag force on a sphere moving towards a corrugated wall," J. Fluid Mech. 513, 247-264 (2004).

[16] A. Mongruel, T. Chastel, E. S. Asmolov, and O. I. Vinogradova, "Effective hydrodynamic boundary conditions for microtextured surfaces," Phys. Rev. E 87, 011002(R) (2013).

[17] T. Chastel and A. Mongruel, "Squeeze-flow between a sphere and a textured wall," Phys. Fluids 28, 023301 (2016).

[18] E. Asmolov, A.V. Belyaev, and O.I. Vinogradova, "Drag force on a sphere moving toward an anisotropic superhydrophobic plane," Phys. Rev. E 84, 026330 (2011).

[19] C. Ybert, C. Barentin, C. Cottin-Bizonne, P. Joseph, and L. Bocquet, "Achieving large slip with superhydrophobic surfaces : scaling laws for generic geometries," Phys. Fluids 19, 123601 (2007). 
[20] N. Lecoq, F. Feuillebois, N. Anthore, R. Anthore, F. Bostel, and C. Petipas, "Precise measurement of particle-wall interactions at low Reynolds number using laser interferometry," Phys. Fluids A 5, 3-12 (1993).

[21] R. G. Cox and H. Brenner, "The slow motion of a sphere through a viscous fluid towards a plane surface ii small gaps widths, including inertial effects," Chem. Eng. Sci 22, 1753-1777 (1967).

[22] O.I. Vinogradova and G.E. Yakubov, "Surface roughness and hydrodynamic boundary conditions," Phys. Rev. E 73, 045302 (2006).

[23] A. Maali, Y. Pan, B. Bushnan, and E. Charlaix, "Hydrodynamic drag-force measurement and slip length on microstructured surfaces," Phys. Rev. E. 85, 066310 (2012).

[24] A. Steinberger, C. Cottin-Bizonne, P. Kleinmann, and E. Charlaix, "High friction on a bubble mattress," Nature materials 6, 665-668 (2007).

[25] T.V. Nizkaya, A.L. Dubov, A. Mourran, and O.I. Vinogradova, "Probing effective slippage on superhydrophobic stripes by atomic force microscopy," Soft Matter 12, 6910-6917 (2016).

[26] J.P. Rothstein, "Slip on superhydrophobic surfaces," Annu. Rev. Fluid Mech. 42, 89-109 (2010).

[27] T.V. Nizkaya, E.S. Asmolov, and O.I. Vinogradova, "Gas cushion model and hydrodynamic boundary conditions for superhydrophobic textures," Phys. Rev. E 90, 043017 (2014).

[28] A. M. J. Davis and E. Lauga, "Hydrodynamic friction of fakir-like superhydrophobic surfaces," J. Fluid Mech 661, 402-411 (2010).

[29] C-O. Ng and C. Wang, "Apparent slip arising from Stokes shear flow over a bidimensional patterned surface," Microfluid Nanofluid 8, 361-371 (2010).

[30] O. Schnitzer and E. Yariv, "Small-solid-fraction approximation for the slip-length tensor of micropillared superhydrophobic surfaces," J. Fluid Mech. 843, 637-652 (2018).

[31] S. Kuwabara, "The forces experienced by randomly distributed parallel cylinders perpendicular to the flow direction at small Reynolds number," J. Phys. Soc. Japan. 14, 527-532 (1959).

[32] J. Yeom, D. D. Agonafer, J. H. Han, and M. A. Shannon, "Low Reynolds number flow across an array of cylindrical microposts in a microchannel and figure-of-merit analysis of micropost-filled microreactors," J. Micromech. Microeng. 19, 065025 (2009). 